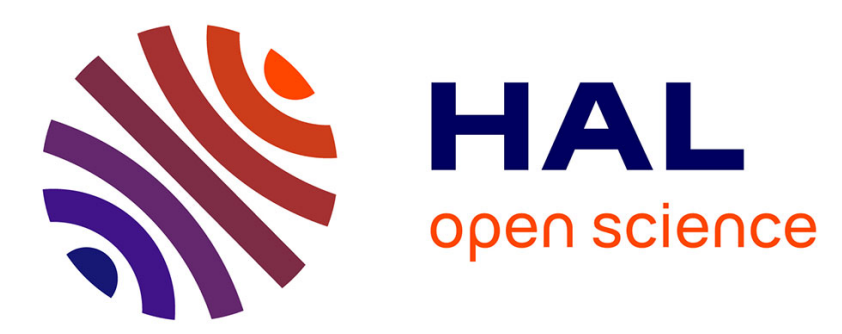

\title{
A 2-dimension dynamic Bayesian network for large-scale degradation modelling with an application to a bridges network
}

\author{
Alex Kosgodagan, Oswaldo Morales-Nápoles, Thomas G. Yeung, Wim \\ Courage, Johan Maljaars, Bruno Castanier
}

\section{To cite this version:}

Alex Kosgodagan, Oswaldo Morales-Nápoles, Thomas G. Yeung, Wim Courage, Johan Maljaars, et al.. A 2-dimension dynamic Bayesian network for large-scale degradation modelling with an application to a bridges network. Computer-Aided Civil and Infrastructure Engineering, 2017, 10.1111/mice.12286 . hal-01517174

\section{HAL Id: hal-01517174 \\ https://hal.science/hal-01517174}

Submitted on 2 May 2017

HAL is a multi-disciplinary open access archive for the deposit and dissemination of scientific research documents, whether they are published or not. The documents may come from teaching and research institutions in France or abroad, or from public or private research centers.
L'archive ouverte pluridisciplinaire HAL, est destinée au dépôt et à la diffusion de documents scientifiques de niveau recherche, publiés ou non, émanant des établissements d'enseignement et de recherche français ou étrangers, des laboratoires publics ou privés. 


\title{
A 2-dimension dynamic Bayesian network for large-scale degradation modelling with an application to a bridges network
}

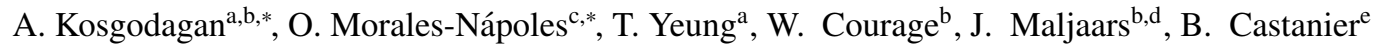 \\ ${ }^{a}$ École des Mines de Nantes, IRCCyN, 4, rue Alfred Kastler, B.P. 20722, F-44307 Nantes Cedex 3, France \\ ${ }^{b}$ Netherlands Organization for Applied Scientific Research, Structural Reliability, Van Mourik Broekmanweg 6, 2628 XE Delft, Netherlands \\ ${ }^{c}$ Faculty of Civil Engineering, Delft Univ. of Technology, 2600 GA Delft, Netherlands \\ ${ }^{d}$ Department of the Built Environment, Eindhoven Univ. of Technology, 5600 MB Eindhoven, Netherlands \\ ${ }^{e}$ Laboratoire Angevin de Recherche en Ingénierie des Systèmes, Université d'Angers, 62 avenue Notre Dame du Lac, 49000 Angers, France
}

\begin{abstract}
Modelling the stochastic evolution of a large-scale fleet or network generally proves to be challenging. This difficulty may be compounded through complex relationships between various assets in the network. Although a great number of probabilistic graph-based models (e.g., Bayesian networks) have been developed recently to describe the behaviour of single assets, one can find significantly fewer approaches addressing a fully integrated network. It is proposed an extension to the standard dynamic Bayesian network by introducing an additional dimension for multiple elements. These elements are then linked through a set of covariates which translate the probabilistic dependencies. A Markov chain is utilized to model the elements and develop a distribution-free mathematical framework to parametrize the transition probabilities without previous data. This is achieved by borrowing from Cooke's method for structured expert judgement and also applied to the quantification of the covariate relationships. Some metrics are also presented for evaluating the sensitivity of information inserted into the covariate-DBN where the focus is given on two specific type of configurations. The model is applied to a real-world example of steel bridge network in the Netherlands. Numerical examples highlight the inference mechanism and show the sensitivity of information inserted in various ways. It is shown that information is most valuable very early and decreases substantially over time. Resulting observations entail the reduction of inference combinations and by extension a computational gain to select the most sensitive pieces of information.
\end{abstract}

Keywords: Dynamic Bayesian network, Structured expert judgment, Covariates, Orthotropic steel bridges, Sensitivity

\section{Introduction}

Degradation modelling in reliability and risk analysis is a topic that has attracted countless pieces of research work. Throughout the past decades, researchers have drawn little attention to fleet- or network-scale problems. More specifically, in the ground transportation infrastructure field, a few recent papers treat bridge networks (?). As one would expect, when considering systems on a much larger scale, the number of variables and uncertainties increases significantly as compared to looking only locally at individual assets. The former approach does not further facilitate cost-efficient strategies

\footnotetext{
${ }^{*}$ Corresponding author

Email addresses: alex.kosgoda@mines-nantes.fr

(A. Kosgodagan), o.moralesnapoles@tudelft.nl

(O. Morales-Nápoles)

Preprint submitted to Elsevier
}

in terms of future maintenance plans at a larger scale. This has become even more desirable with the growing use of continuous monitoring that asset managers may use to either update the current knowledge of a system or formulate predictions on various key indicators. In the reliability field many different type of assets are continuously and efficiently monitored (e.g., roads, buildings, bridges, etc.), however it is often cost-prohibitive and not vital to place a monitoring installation at each individual asset. By consequence, collected data varies in size and informativeness from asset to asset so that much effort is often given to identifying the most relevant and sensitive elements.

Particularly for deterioration modelling, uncertainty surrounding the degradation process is highly present from environmental conditions, material properties, etc 
for instance. Markov-based models are now widely accepted as suitable stochastic processes especially in the bridge degradation modelling domain (?). It is common practice to exploit inspection data on various parts of an asset to model both the component-level and the overall condition through Markov processes. The main task in Markov-based models reduces almost exclusively to the assessment of the transition probabilities. Several general methodologies have been developed to using condition ratings data as well as those specific to bridges (????). In the case where condition ratings are not available, synthetic condition states can be sampled from assumed prior distributions or degradation models. In particular, in ?, condition state values are randomly generated to represent a range of condition states at each ten-year interval using Weibull distribution and a Latin hypercube simulation. However the degradation pattern comes from knowledge of the specific area of concern or is somewhat assumed a priori like in ? where a hazard exponential model is used to derive the Markov transition probabilities. While almost the entire literature encourages the use of either the two methodologies mentioned, there is a scarcity of models investigating the case where very limited field data are to be used.

The objective of this paper is to model the degradation for a network of "similarly classified" assets under very limited data. It is denotes "similarly classified" assets as those state evolutions are highly correlated. A new methodology is proposed to parametrize the transition probabilities of a Markov chain of a particular asset. In absence of the aforementioned data, or where data is very limited, it is proposed a method to quantify the mean duration of the first passage time between degradation conditions to derive the transition probabilities through a simple linear equation. The expected durations of transitions are elicited by means of the classical Cooke's method (?) for combining expert opinions. This provides a procedure that fully quantifies in a probabilistic way durations of transition. Furthermore, Cooke's method also allows us to provide a distributionfree method in order to obtain the transition probabilities. To our knowledge, this is the first application of Cooke's method to parametrize a Markov chain.

Information on underlying mechanisms (covariates) interacting with one another may be available for some of the most relevant elements. Their role is twofold: (1) they serve as factors impacting degradation upon which the Markov process depends and (2) to generate a coherent probabilistic framework to address dependency among assets in the network-scale problem. Multi-dimensional (e.g., spatial) dependencies that may exist in the network elements are conveyed through these covariates. The new methodology proposed in this paper extends the classic framework of dynamic Bayesian networks (DBNs) by providing an approach to model the state of a large-scale set of assets in a consistent manner without necessary data for the standard parametrization approaches. The extended DBN, which is termed a covariate- $D B N$, also allows the propagation of new information from assets for which data is available into others for which data may be limited. The conditional probabilities of the DBN are also derived using the structured expert judgment (SEJ) approach described above for the Markov chain.

BNs have been extensively used in reliability and civil engineering where high-dimensional probabilistic evaluation is necessary. For discrete BNs, the quantitative burden related to both the quantification of conditional probability assessments and the inference mechanism are known to be the main limitations. ?? introduce a high-dimensional probabilistic model using BNs for safety and risk analysis in the railway domain where 7,820 variables (on separate BNs) have been used. ? proposed a DBN model for probabilistic assessment of tunnel construction performance including a modified version of the Frontier algorithm to perform inference. One of the advantages shown in each of the three above-cited articles is that BNs can be a powerful tool to quantify the risk of extraordinary events. In this paper it is provided a global methodology through the so-called covariate-DBN model for asset management. Computationally-wise, it is shown that the inference combinations can significantly be reduced by advantageously exploiting results regarding the sensitivity of unexpected events.

The use of embedded covariates in a DBN suggests an analogy with Markov switching models (?) as they were introduced to model this type of stochastic process by adding conditionality through either observed or unobserved variables. These types of models were extensively developed in econometrics and finance whose main purpose is to capture switching regimes of time series data. The method's purpose here is, however, not to model changes in time series switching regimes but rather covariates are introduced with the twofold above-mentioned role. Secondly, modelling degradation through observable covariates also relates to the work of ? and ? in survival analysis. Deterioration dynamics is driven by continuous stochastic processes and covariates in both approaches, however, they do not address multi-dimensional distributions as is done through a DBN.

In a very recent paper by ?, they develop a DBN approach including nodes representing spatial dependency 
across different location for revealing trophic dynamics in fisheries ecology. However, the proposed framework is specific to the application considered through spatial nodes and thus does not offer a general methodology to address classes of problems discussed above. Moreover, it is emphasized that the spatial characteristic may not be a systematic factor to generate the network. One could also think of other links found between multiple elements, such as common material properties, relationships between physics-based phenomena, etc.

The remainder of the paper is organized as follows. The following section presents the degradation process framework combining a discrete Markov process with a DBN where the two-dimensional network-scale extension is presented. First, the procedure of estimating the transition probabilities is detailed. Second, how its dynamics is influenced by covariates and the networkscale DBN is formulated. Section 3 introduces the SEJ method to calibrate the Markov transition probabilities and conditional probabilities in the covariate-DBN model. Section 4 highlights a bridge network deterioration example based on traffic and load as selected covariates. Section 5 provides numerical experimentation highlighting the benefits of inference throughout the covariate-DBN model before drawing final conclusions and providing perspectives on future work.

\section{Deterioration framework}

A finite discrete-time Markov stochastic process $\left\{D_{t}^{(k)}, t \geq 0\right\}$ is used to model the degradation for element $k$. Whenever possible, it will be omitted superscript $(k)$ for every stochastic process. The goal is simply to describe the probability that each of the elements can be in a particular state at time $t$ conditionally on the previous state and some selected covariates. covariates are used to represent observable random variables that influence the degradation process $\left\{D_{t}\right\}$. To address the network-scale issue, an extension of the classic Dynamic Bayesian network (DBN) framework is presented.

\subsection{Markov Chain}

Discrete-time Markov processes have been extensively used in the context of risk, reliability and maintenance management for civil infrastructures (??). The Markov property mainly characterizes this class of stochastic processes. Recall that this property stipulates that it is only needed to know where the process $D_{t}$ stands at present time $t$ (first order), as opposed to rely on its complete history, to predict in a probabilistic sense how the process behaves in the future. It is denoted by $\{1, \ldots, \Omega\}$ the set in which $D_{t}$ takes values. Conventionally, it is written the one time step transition probability $p_{i, j}$ from state $i$ to $j, i, j \in\{1, \ldots, \Omega\}$, the probability $P\left(D_{t+1}=j \mid D_{t}=i\right)$. In the present case it is assumed a sequential degradation, meaning that only the $p_{i, i}, p_{i, i+1}>0$ with $p_{i, i}+p_{i, i+1}=1$. As it is assumed that bridges are in the best condition when newly constructed, $P\left(D_{0}=1\right)=1$. The stochastic process $\left\{D_{t}, t \geq 0\right\}$ that models degradation is usually defined by the (Chapman-Kolmogorov) equation

$$
P\left(D_{t}=j \mid D_{0}=1\right)=\mathbf{P}^{t}(1, j)
$$

where $\mathbf{P}^{t}$ is the transition probability matrix (TPM) to the power $t$ and $\mathbf{P}^{t}(1, j)$ refers to row 1 and column $j$ of $\mathbf{P}^{t}$, with $1 \leq j \leq \Omega$ and for every $t \geq 0, \sum_{j} P\left(D_{t}=\right.$ $\left.j \mid D_{0}=1\right)=1$. A set of $n \geq 1$ so-called covariates is further introduced which designate random variables denoted by $\Theta_{t}=\left(\theta_{1, t}, \ldots, \theta_{n, t}\right)$ for each time step $t$, with $\left(\theta_{1, t}, \ldots, \theta_{n, t}\right) \in C_{1} \times \cdots \times C_{n}$, so that the process $\left\{D_{t}\right\}$ is dynamically influenced by such quantities. The transition probabilities are thus given by

$$
\begin{gathered}
p_{i, j}=\sum_{c_{1}, \ldots, c_{n}} P\left(D_{t}=j \mid D_{t-1}=i, \theta_{1, t}=c_{1}, \ldots, \theta_{n, t}=c_{n}\right) \\
\times P\left(\theta_{1, t}=c_{1}, \ldots, \theta_{n, t}=c_{n}\right)
\end{gathered}
$$

Covariates may either directly or indirectly impact $\left\{D_{t}\right\}$. An indirect covariate would impact another covariate rather than directly $D_{t}$. This is precisely the reason why Bayesian networks are used as a suitable framework to handle the dependence structure and make transparent its visualization and quantification. The latter is introduced in the section 2.3 where the complete definition of the new DBN framework is presented.

\subsection{Bayesian networks}

Bayesian networks (BNs) are probabilistic graphs of relative recent development as their formalism was put forward by ?. A compact way of defining BNs can be divided into two parts, namely graphical and probabilistic denoted by the couple $(\mathcal{G}, \mathcal{P})$. Often those are described as the qualitative and quantitative features, respectively. A BN is a directed acyclic graph (DAG) and $\mathcal{G}=(\mathcal{N}, \mathcal{E})$, where sets $\mathcal{N}$ and $\mathcal{E}$ respectively represent nodes and arcs or arrows. Elements of $\mathcal{E}$ will be expressed as $(a ; b)$ whenever $\{a\}$ is incident to $\{b\}$. In probabilistic terms, nodes are univariate random variables and arcs translate probabilistic dependencies. The other part $\mathcal{P}$ refers thus to a probabilistic characterization through conditional distributions associated to $\mathcal{G}$ 
between (sets of) nodes linked by the arcs. The complete characterization of a $\mathrm{BN}$ reduces solely to the assessment of the couple $(\mathcal{G}, \mathcal{P})$.

A BN encodes the probability density or mass function on a set of variables $\mathbf{X}=\left\{X_{1}, \ldots, X_{N}\right\}$ by specifying a set of conditional independence statements in the DAG associated with a set of conditional probability functions. It thus provides a representation of a high dimensional probability distribution on $\mathbf{X}$. The joint probability mass function $f_{X_{1}, \ldots, X_{N}}$ over variables $\left\{X_{1}, \ldots, X_{N}\right\}$ can be expressed as

$$
f_{X_{1}, \ldots, X_{N}}=\prod_{i=1}^{N} f_{X_{i} \mid p a\left(X_{i}\right)}
$$

where $p a\left(X_{i}\right)$ denotes the set of parents for node $X_{i}$. Distributions can be discrete, continuous or a mixture of both. However in this paper it is exclusively made use of the discrete case. It is referred to ? and ? for a broader survey on the remainder classes.

In the same manner it is denoted by $\mathbf{X}$ the set of $n$ variables $\left\{X_{1}, \ldots, X_{n}\right\}, \mathbf{Y}$ and $\mathbf{Z}$ designate sets of $n$ variables $\left\{Y_{1}, \ldots, Y_{n}\right\}$ and $\left\{Z_{1}, \ldots, Z_{n}\right\}$ respectively. Furthermore, it is understood the notation $\mathbf{X} \perp \mathbf{Y} \mid \mathbf{Z}$ to suggest that $\mathbf{X}$ and $\mathbf{Y}$ are conditionally independent given $\mathbf{Z}$ which is equivalent to write $\mathbf{X} \not \mathbf{Y}$ saying that $\mathbf{X}$ and $\mathbf{Y}$ are marginally dependent. Likewise, it is used $\mathbf{X} \not \perp \mathbf{Y} \mid \mathbf{Z}$ to make explicit that $\mathbf{X}$ and $\mathbf{Y}$ are conditionally dependent given $\mathbf{Z}$. As briefly mentioned before, a remarkable property coming from graph theory that BNs possess is called the directional separation (d-separation). The latter transcribes a graphical attribute corresponding to three distinct visual layouts into probabilistic (in)dependence statements between sets of variables. The cases of concern regarding the d-separation property are summarized as follows:

- $\mathbf{X} \leftarrow \mathbf{Y} \rightarrow \mathbf{Z}$; this means that $\mathbf{X}$ is independent of $\mathbf{Z}$ given $\mathbf{Y}(\mathbf{X} \perp \mathbf{Z} \mid \mathbf{Y})$, however $\mathbf{X}$ and $\mathbf{Z}$ are not marginally independent $(\mathbf{X} \not \mathbf{Z})$

- $\mathbf{X} \rightarrow \mathbf{Y} \rightarrow \mathbf{Z}$; this layout has the same interpretation as the previous one

- $\mathbf{X} \rightarrow \mathbf{Y} \leftarrow \mathbf{Z}$; in this case, we have $\mathbf{X} \not \mathbf{Y} \mid \mathbf{Z}$ implying $\mathbf{X} \perp \mathbf{Y}$

Another feature which makes BNs attractive is symbolized by the ability to perform inference. More specifically, the network can be used to update the probability distribution over the state of a subset of variables when information becomes available for other variables. Regarding the foundations of exact inference mechanism, we refer again to ?. Exact inference in the discrete case is an NP-hard problem meaning that the complexity is exponential in the number of states and degree ${ }^{1}$ of nodes. Hence, approximation algorithms like variational or Monte Carlo methods emerged in order to mitigate the computational burden for high dimension and complex BNs. Readers are referred to ? for a large review on probabilistic inference for graphical models. In the bridge engineering field, information can stem from inspection data, crack measurement testing or even monitoring systems collecting inputs regarding traffic as shown in section 4 .

In a static discrete $\mathrm{BN}$, nodes stand for discrete random variables which are the most common version that have been developed in risk and reliability modelling (?). The BN displayed in Fig. 1 shows how the set $\Theta$ of four time-independent covariates, namely $\Theta=$ $\left(\theta_{1}, \theta_{2}, \theta_{3}, \theta_{4}\right)$, and state node $D$ can be linked when not accounting for any time nor network dimensions. In this example, nodes $\theta_{2}$ and $\theta_{4}$ are directly connected to $D$. Nonetheless, a more suitable version in the present case refers to dynamic BNs accounting for time dynamics through the process $\left\{D_{t}\right\}$ which is presented in the next section.

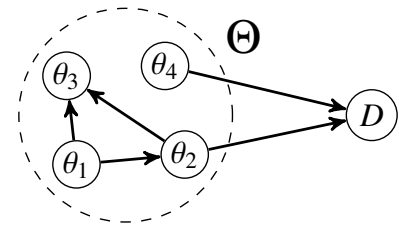

Figure 1: Static covariate-BN structure

\subsection{Covariate- $D B N$}

While BNs are useful for modelling a dependence structure among random variables, they do not capture the evolution over time. For modelling dependencies between stochastic processes by direct or indirect covariates as described above in eq. (2), a convenient tool is Dynamic Bayesian networks (DBNs). Especially in degradation modelling, DBNs are a well suited (?). Time is represented as a discrete time slices or steps which are connected by directed arcs from nodes in slice $t$ to nodes in slice $t+1$. Note that the network structure is identical in each slice (i.e., does not change over time). A DBN that contains time-dependent conditional distributions is denoted a non-homogeneous DBN. Furthermore, the dependence between the deterioration nodes

\footnotetext{
${ }^{1}$ the degree of a node is understood by the number of edges incident to it
} 
is in compliance with the Markovian property. Only time slice $t$ is dependent on time slice $t+1$; thus, only current information is required to assess the probabilistic evolution (i.e, it is memoryless). Like the static version, the characterization of a DBN is defined by the graph structure at time $t$, between $t$ and $t+1$, and the assessment of the conditional distributions for $t=0$ and between slices $t$ and $t+1$. Similar to the static BNs, inference may also be performed and there have been specific algorithms developed for DBN frameworks (?).

it is proposed an extension of the classic DBN formulation to a fleet- or network-level through the covariates introduced above. Network covariates make use of relationships between one or several elements composing the network. These could stand, for instance, for operating and environmental conditions, structure characteristics, material properties, etc. While data may be unavailable for the key metric of interest (i.e., $D_{t}$ ), information on various covariates may be obtained. These covariates can then be used as a means to insert information that will be propagated throughout the network due to their dependence structure with state of interest $D_{t}$. This extends the traditional DBN which contains only time dependence to additional dependence dimensions. In our model this second dimension is conveyed by the covariates.

Let $\Theta_{t}^{(k)}=\left(\theta_{1, t}^{(k)}, \ldots, \theta_{n, t}^{(k)}\right)$ be the set of $n$ covariates at time $t$ for element $k$ of the network. Note the addition of superscript $k$ for the interdependent network case. A visual representation example of the extended DBN model is reported in Fig. 2. It contains $n=4$ covariates per time slice $t$ for a network composed of two elements $k=\{1,2\}$. The set of covariates for each element $k=\{1,2\}, \Theta_{t}^{(1)}$ and $\Theta_{t}^{(2)}$ is represented by the big dashed circles. It is assumed that in our proposed extended DBN the dependence structure does not change over time, but may change between elements $k$. Covariates may evolve independently or depend on other covariates and may or may not directly impact $\left\{D_{t}\right\}$. This is shown with $\theta_{4, t}^{(k)}$ being independent of $\left(\theta_{1, t}^{(k)}, \theta_{2, t}^{(k)}, \theta_{3, t}^{(k)}\right)$ with $k=1,2$. Precisely, for element 1 the covariates $\theta_{2, t}^{(1)}$ and $\theta_{4, t}^{(1)}$ are directly impacting $\left\{D_{t}^{1}\right\}$ whereas for element 2 , $\theta_{1, t}^{(2)}$ and $\theta_{4, t}^{(2)}$ are playing this role. Again, once this structure is set for each element it is kept over the whole time horizon. Although not shown in Fig 2, for a given element, covariates could also have a time-varying distribution. The latter has already been introduced in ?, but without incorporating a second dimension as is done. The connexions across the different elements are thus made through the set of covariates $\Theta_{t}^{(k)}$. It is also shown in Fig. 2 that $\theta_{1, t}^{(k)}$ and $\theta_{4, t}^{(k)}$ are the covariates perform- ing the linking task. It is assumed that each element has the same set of covariates $\Theta_{t}^{(k)}$, although the dependence structure between covariates of different elements may vary according to the data. The DBN structure can be generalized similarly to what characterizes a classic DBN. For time epoch $0 \leq t \leq S$ and network element $1 \leq k \leq K$, there must be specified:

- the covariate dependence structure for each element $k$ denoted by $\mathcal{G}_{\Theta}^{(k)}=\left(\mathcal{N}_{\Theta}^{(k)}, \mathcal{E}_{\Theta}^{(k)}\right)$ with $\mathcal{N}_{\Theta}^{(k, t)}=$ $\left\{\Theta_{t}^{(k)}\right\}, \mathcal{E}_{\Theta}^{(k)}=\left\{\left(p a\left(\theta_{i, t}^{(k)}\right) ; \theta_{i, t}^{(k)}\right), 1 \leq i \leq n\right\}$ and its set of conditional distribution functions $\mathcal{P}_{\Theta}^{(k)}=$ $\left\{f_{\theta_{i, t}^{(k)} \mid p a\left(\theta_{i, t}^{(k)}\right)}, 1 \leq i \leq n\right\}$

- the covariate-to-element dependence structure denoted by $\mathcal{G}_{D^{\downarrow \Theta}}^{(k)}=\left(\mathcal{N}_{D^{\downarrow}}^{(k)}, \mathcal{E}_{D^{\downarrow \Theta}}^{(k)}\right)$ with $\mathcal{N}_{D^{\downarrow \Theta}}^{(k)}=$ $\left\{D_{t}^{(k)}\right\}, \mathcal{E}_{D^{\downarrow \Theta}}^{(k)}=\left\{\left(p a\left(D_{t}^{(k)}\right)^{\downarrow \Theta_{t}^{(k)}} ; D_{t}^{(k)}\right)\right\}$ and set of conditional distribution functions $\mathcal{P}_{D^{\downarrow \Theta}}^{(k)}=$ $\left\{f_{D_{t}^{(k)} \mid p a\left(D_{t}^{(k)}\right)^{\downarrow \Theta_{t}^{(k)}}}\right\}$ where $p a(X)^{\downarrow \mathbf{Y}}$ designate the set of parents for node $X$ restricted to node set $\mathbf{Y}$.

- the element-to-element dependence structure denoted by $\mathcal{G}_{\Theta}^{(\rightarrow)}=\left(\mathcal{N}_{\Theta}^{(\rightarrow)}, \mathcal{E}_{\Theta}^{(\rightarrow)}\right)$ with $\mathcal{N}_{\Theta}^{(\rightarrow)}=\left\{\Theta_{t}^{(k)}: 1 \leq k \leq K\right\}, \quad \mathcal{E}_{\Theta}^{(\rightarrow)}=$ $\left\{\left(p a\left(\theta_{i, t}^{(k)}\right) ; \theta_{i, t}^{(k)}\right): p a\left(\theta_{i, t}^{(k)}\right) \not \subset \Theta_{t}^{(k)}, 1 \leq i \leq n\right\}$ and conditional probability set $\mathcal{P}_{\Theta}^{(\rightarrow, t)}=$ $\left\{f_{\Theta_{t}^{(k)} \mid p a\left(\theta_{i, t}^{(k)}\right)}: p a\left(\theta_{i, t}^{(k)}\right) \not \subset \Theta_{t}^{(k)}, 1 \leq i \leq n\right\}$

The complete covariate-DBN can now be defined for time horizon $S$ and bridges network size $K$ as $\mathbf{B}^{K, S}=$ $\left\{\mathcal{G}^{K, S}, \mathcal{P}^{K, S}\right\}$ where $\mathcal{G}^{K, S}, \mathcal{P}^{K, S}$ are summarized, respectively, through each of the graph and probabilistic sets introduced above.

\subsection{Network Sensitivity Analysis}

It is proposed a methodology for evaluating the sensitivity of covariate information inserted into the network at different points in both time $t$ and dimension $k$. This aids identifying the key elements of the network, the types of information with the greatest impact, and when and where to observe the network in order to obtain said information. Recall that the set of covariates $\Theta_{t}^{(k)}=\left(\theta_{1, t}^{(k)}, \ldots, \theta_{n, t}^{(k)}\right)$ takes values in $C_{1} \times \cdots \times C_{n}$. Let $\omega_{\Theta}=\left(\omega_{\theta_{j, t}^{(k)}}\right)_{\substack{1 \leq j \leq n \\ 0 \leq t \leq S \\ 1 \leq k \leq K}}$ be the $n$-by- $S$-by- $K$ matrix of one possible combination where each $\omega_{\theta_{j, t}^{(k)}} \in C_{j} \cup N O I$, represents the possible information that can be inserted 


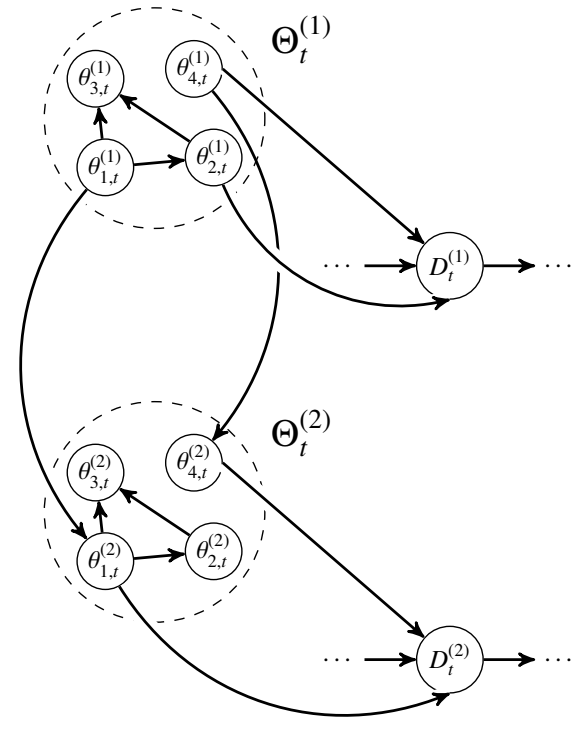

Figure 2: A two-element Covariate-DBN with 4 covariates at time $t$

adding the "no information (NOI)" state. The unconditional case is simply the matrix $\omega_{\Theta}$ with all entries being NOI. The total number of possible combinations of injecting evidence for the covariate-DBN model is given by all the permutations among the set $\left\{C_{1}, \ldots, C_{n}\right\}^{K S}$ given by

$$
e_{\Theta}=\left(\left(\left|C_{1}\right|+1\right) \times \cdots \times\left(\left|C_{n}\right|+1\right)\right)^{K S}-1
$$

with $T$ being the time horizon, $K$ the total number of elements and $\left|C_{j}\right|$ the cardinality of each set $C_{j}, j=$ $1, \ldots, n$. One way to measure the value of the propagated information is to check how much it affects the posterior probability distribution. Fix bridge $0 \leq k \leq K$ and degradation state $i \in\{1, \ldots, \Omega\}$, the following sensitivity measure can therefore be computed

$$
\sigma_{i, \Theta}=\frac{\left|P\left(D_{t}^{(k)}=i\right)-P\left(D_{t}^{(k)}=i \mid \omega_{\Theta}\right)\right|}{P\left(D_{t}^{(k)}=i\right)}
$$

This metric may provide insight on when and for what duration new information should be obtained as well as the quantity and location deployed across the network. Let $\tau=\inf \left\{t \geq 0: \forall j, k, \omega_{\theta_{j, t}^{(k)}} \neq N O I\right\}$, therefore

$$
\sigma_{i, \Theta} \begin{cases}=0 & \text { if } t<\tau \\ >0 & \text { otherwise }\end{cases}
$$

This means that the earliest piece of evidence being inserted only impacts the posterior probabilities of $P\left(D_{t}^{(k)}=i \mid \omega_{\Theta}\right)$ for $t>\tau$.
To study how sensitive the network reacts, it is prohibitive to cover the list of all possibilities as $e_{\Theta}$ grows exponentially along $K$ and $S$. Two different types of configurations are put forward to gain insight from a large covariate space: 1) the effect of information being inserted individually at different points in time and 2 ) the cumulative effect of inserting information at multiple points in time. The study is further restricted to the case where only the same type of information is entered over time.

For a fixed covariate $j \in\{1, \ldots, n\}$, covariate value $c \in$ $C_{j}$, and element $k \in 1, \ldots, K$, let $\mu_{\omega}^{(k)} \in\{0, \ldots, S\}$ be the time a single piece of information $\left(\omega_{\theta_{j, t}^{(k)}}\right)_{0 \leq t \leq S}$ is inserted into the network. Furthermore, let $\eta_{\omega}^{(k)} \in\{0, \ldots, S\}$ be the time up to which consecutive pieces of information are inserted beginning at $t=0$. Then the matrix $\omega_{\Theta}=$ $\left(\omega_{\theta_{j, t}^{(k)}}\right)_{\substack{0 \leq t \leq S \\ 1 \leq k \leq K}}$ can be a function of $\eta_{\omega}^{(k)}$ and the binomial coefficient $\left(\begin{array}{c}S \\ n^{(k)}\end{array}\right)$ which gives all possible orderings for a specific number of pieces of evidence. Thus we obtain

$$
\frac{d \sigma_{i, \Theta}}{d \eta_{\omega}^{(k)}} \begin{cases}=0 & \text { if } t \geq \tau \\ >0 & \text { otherwise }\end{cases}
$$

This shows that for a specific element $k$ and a certain covariate $\theta_{j, t}$, regardless of the way pieces of information are incorporated, i.e. the various permutations among the set $C_{j}, \sigma_{i, \Theta}$ increases or is constant along $\eta_{\omega}^{(k)}$. This result holds for cumulative information incorporated across different elements. This results is particularly desirable in the reliability domain as it highlights the usefulness to obtain field data in a temporal cumulative manner from a specific element or several of them. Not only does it primarily impact its own posterior distribution but it additionally affects the probability of the other elements. The sensitivity value (5) facilitates the quantitative identification of elements in the network with minor consequence on others and thus reduce the need of observation.

\section{Parametrization through Structured Expert Judgment}

The goal here is to parameterize the transition probabilities of the Markov chain $D_{t}$. The classical SEJ model developed by ? is used which is a performance-based weighted averaging model to aggregate individual experts distributions into a single combined one. It is both a widely accepted (?) and appropriate method when quantitative data is missing, of dubious quality, or is insufficient for obtaining desired outcomes. 


\subsection{Cooke's model for eliciting expert opinions}

The protocol of ? was followed which provides a clear statement of the questions to be answered, documents critical underlying assumptions, and establishes a logical structure for the elicitation interview. Experts are asked to specify their quantiles (e.g., 5th, 50th and 95th) of an uncertainty distribution regarding variables of interest and seed variables tailored to the problem considered. Seed variables are known quantities used to compute two measures of performance of the experts: the calibration and information scores. Loosely, calibration measures the statistical likelihood that a set of experimental results correspond, in a statistical sense, with the experts assessments. Information measures the degree to which a distribution is concentrated. The weights are derived from experts calibration and information scores, as measured on seed variables. Seed variables serve a threefold purpose:

(i) to quantify experts performance as subjective probability assessors

(ii) to enable performance-optimized combinations of expert distributions

(iii) to evaluate and hopefully validate the combination of expert judgments.

\subsection{Calibration of $p_{i, j}$}

Several developments must be made in order to apply the Cooke's method to parametrize a Markov chain. Instead of explicitly eliciting $p_{i, j}$ expected transition time between consecutive states $i$ to $i+1$ are asked. Directly estimating probabilities should be avoided as performing such a task is known to be challenging. Quantities which experts are more familiar with are thus recommended to further derive the ones of interest. In order to quantify $p_{i, j}$ introduced in eq.(2), the expected time it takes for a bridge to transit between states $i$ and $j$ is given by

$$
E\left[T_{i, j}\right]=1+\sum_{k \neq j} E\left[T_{k, j}\right] p_{i, k}
$$

where $T_{i, j}=\inf \left\{M: D_{M}=j, D_{M-1} \neq j, \ldots, D_{m+1} \neq\right.$ $\left.j \mid D_{m}=i\right\}$ is a strictly positive integer random variable and represents the first passage time from state $i$ to state $j$, with $0 \leq m<M$. When $j=i$ we have $E\left[T_{i, i}\right]=1 / \pi_{i}$, where $\pi_{i}$ is the limit distribution of the Markov chain for state $i, \lim _{t \rightarrow+\infty} P\left(D_{t}=i\right)=\pi_{i}$. Typically, as state $\{\Omega\}$ is the only absorbing state, $\pi=\left(\pi_{1}, \ldots, \pi_{\Omega}\right)=(0, \ldots, 1)$ so $E\left[T_{i, j}\right]=\infty, \forall i \geq j$. In the very general case where $\mathbf{P}$ is complete, i.e., when interventions improving the state of an element are allowed, and the expected transitions of first passage time matrices are given respectively by

$$
\begin{aligned}
\mathbf{P} & =\left(\begin{array}{ccc}
p_{1,1} & \ldots & p_{1, \Omega} \\
\vdots & \ddots & \vdots \\
p_{\Omega, 1} & \ldots & p_{\Omega, \Omega}
\end{array}\right) \\
\text { and } \quad \mathbf{E} & =\left(\begin{array}{ccc}
E\left[T_{1,1}\right] & \ldots & E\left[T_{1, \Omega}\right] \\
\vdots & \ddots & \vdots \\
E\left[T_{\Omega, 1}\right] & \ldots & E\left[T_{\Omega, \Omega}\right]
\end{array}\right)
\end{aligned}
$$

from eq. (8), the following linear system of equations has to be solved

$$
\mathbf{P}^{*}(\mathbf{E}-\operatorname{diag}(\mathbf{E}))=\mathbf{E}-\mathbf{1}
$$

where $*$ is the usual matrix product operator, $\operatorname{diag}(\mathbf{E})$ is the matrix having the values $E\left[T_{i, i}\right]$ and zeros in each of the other entries and $\mathbf{1}$ is the matrix having ones in every entry. For matrix $\mathbf{E}$, the entry $(i, j$ ) (with $i \neq j$ ) is non infinite if there exists $M>0$ such that for any $m, 0 \leq$ $m<M, P\left(X_{M}=j \mid X_{m}=i\right)>0 \Leftrightarrow \mathbf{P}^{M-m}(i, j)>0$. The latter simply translates quantitatively the fact there must exist a path starting from state $i$ to reach state $j$ in order to have a finite (expectation of) first passage time. Recall that $p_{i, i}+p_{i, i+1}=1$ so only the $p_{i, i}$ or $p_{i, i+1}$ need be specified. The case of concern which features sequential degradation behaviour entails that from eq. (9), for each $i \in\{1, \ldots, \Omega\}$, we have

$$
p_{i, i}=1-\frac{1}{E\left[T_{i, i+1}\right]}
$$

so that $\Omega-1$ expected transitions have to be elicited. From eq. (10), $E\left[T_{i, i+1}\right] \geq 1$ otherwise it yields $p_{i, i}<$ 0 . If an expert gives an estimate where $E\left[T_{i, i+1}\right]<1$, one can simply rescale the time step to a smaller time unit. Parametrizing the model amounts to calibrating the quantities $P\left(D_{t}=j \mid D_{t-1}=i, \theta_{1, t}=c_{1}, \ldots, \theta_{n, t}=c_{n}\right)$ (eq. (2)) and $E\left[T_{i, i+1}\right]$ (eq. (10)), as the joint distribution $P\left(\theta_{1, t}=c_{1}, \ldots, \theta_{n, t}=c_{n}\right)$ is assumed to be empirically obtained. The two main questions are then generated as follows

$Q 1$ "Could you provide the $5^{\text {th }}, 50^{\text {th }}, 95^{\text {th }}$ quantiles of your uncertainty distribution about the expected years that it takes for each of the $K$ elements considered to transit between each of the states in $\{1, \ldots, \Omega\}$ ?"

Q2 "Consider a sample of 100000 data points each representing the following event. At time $t-1$ a certain element $k$ was in a certain condition state 
$(1, \ldots, \Omega)$ and the covariates directly incident to the process $D_{t}^{(k)}$ were observed to be in each their possible states (i.e., cardinality of the state space of incident covariates). Recall that it is assumed elements can only deteriorate to their next worse state or remain in the same state at the next time step. Out of these 100000 samples, what is the number of these assets transitioning to their next worse state at the next time step ?"

Note that Q1 and Q2 must be elicited for each element $k$, thus the number of questions to be asked is $2 k$. However, the total network size may be dramatically increased while limiting $k$, by considering different classes in which multiple elements belong to the same class. This would create classes of i.i.d. elements. More precisely, the variable $\kappa$ is introduced as to represent the number of classes of same type of assets, each containing a certain number of elements in each set $\gamma_{i}, i=1, \ldots, \kappa$ such that $K=\sum_{i=1}^{\kappa}\left|\gamma_{i}\right|$. Whenever possible and in order to lighten the burden of notations, the $\kappa$ index can be omitted as the derived results do not benefit additionally from this. The elicitation complexity of our proposed model is derived from the number of questions that need to be asked, however, a very large network can be constructed without needing to elicit responses for each element if they are of the same class. This will be detailed in the following sections of our bridge application in which hundreds of bridges may be present but only a few classes. In such a context, only questions on the classes need be elicited and not each individual bridge in the network. This further highlights the limited data framework application of this model.

The covariate-DBN methodology is summarized through the diagram displayed in Fig. 3. The arrows from the $S E J(Q 2)$ node pointing to eq (2) is more precisely referring to member $P\left(D_{t}=j \mid D_{t-1}=i, \theta_{1, t}=\right.$ $c_{1}, \ldots, \theta_{n, t}=c_{n}$ ) making the one-to-one correspondence link between the covariates $\Theta_{t}^{(1)}, \ldots, \Theta_{t}^{(K)}$ and the Markov processes $\left\{D_{t}^{(1)}\right\}, \ldots,\left\{D_{t}^{(K)}\right\}$. The latter are specified by the expectation of the random variable $T_{i, i+1}$ (eq. (10)) which is parametrize from SEJ by Q1. The dashed double-oriented arrow among the covariate sets refers to the possible dependence relationships between them. Note that the Fig. 3 only represents one slice in time, therefore the $t$ subscript has been omitted. To represent the total time horizon, Fig. 3 would be repeated for all $t \in\{0, \ldots, S\}$.

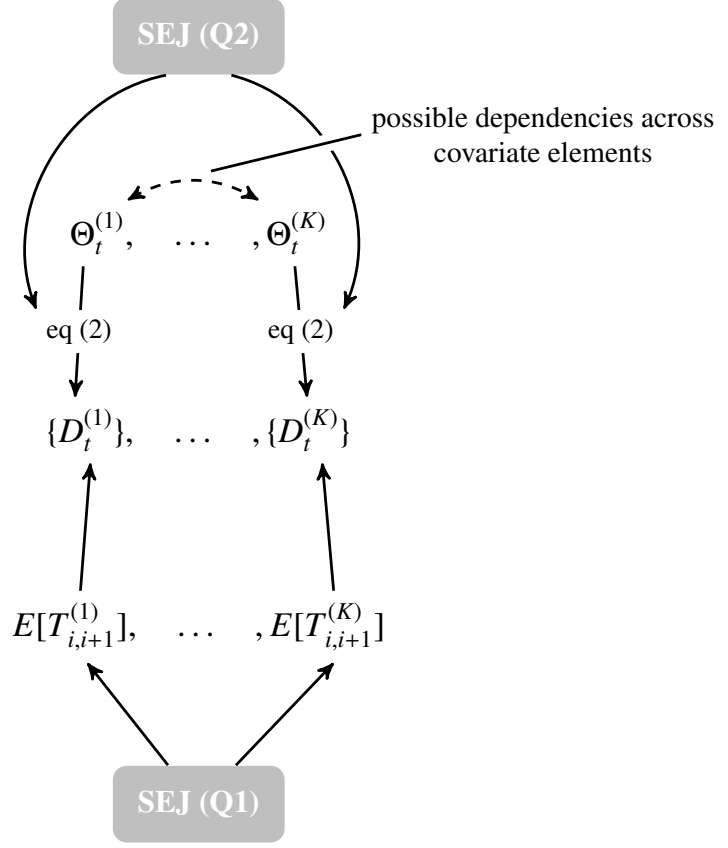

Figure 3: Diagram of the covariate-DBN methodology

\section{Bridge Network Application}

This section treats degradation modeling for a network of motorway steel bridges. Two different classes of motorway bridges are specifically considered with a steel (so-called orthotropic) bridge deck, namely moveable and fixed. On the network of motorways in the Netherlands there are approximately 100 steel bridges, divided into movable and fixed types (?). These types should be quite representative of the category encompassing motorway steel bridges located in the Dutch bridge network. A key characteristic of a bridge is its deck plate thickness. The thickness of the bridges may vary throughout the network. It is assumed that the deck plate thickness for moveable and fixed bridge is chosen to be $12 \mathrm{~mm}$ and $10 \mathrm{~mm}$, respectively. Furthermore, the thickness and type of deck plate overlay are assumed a $6 \mathrm{~mm}$ thick epoxy overlay and a $100 \mathrm{~mm}$ asphalt is applied for moveable and fixed bridges, respectively.

The underlying physical deteriorating process considered here is fatigue crack growth in the bridge deck which occurs due to repetitive loading by vehicles' axles. Fatigue is a degeneration process developing in time such that it can be detected before they grow so large that they obstruct the safe use or even integrity of the structure. It is assumed that the crack growth rate decreases for increasing deck plate thickness and surface finish. By consequence, the covariates chosen are 
traffic and loading as they are the main endogenous contributors in this mechanism. Data coming from a monitoring system located in the Netherlands is available, presented subsequently and used to evaluate some of the conditional probability distribution sets.

\subsection{Dependence structure}

Traffic and loading covariates are denoted by $\left\{T_{t}^{(k)}\right\}$ and $\left\{L_{t}^{(k)}\right\}$, respectively. Thus, $\Theta_{t}^{(k)}=\left(T_{t}^{(k)}, L_{t}^{(k)}\right)$, for any bridge $k$. The typical dynamic dependence structure for the deterioration of any bridge $k$ is sequential, that is, $T_{t}^{(k)} \rightarrow L_{t}^{(k)} \rightarrow D_{t}^{(k)}$ for any time slice $t$. The edges connecting successively the degradation nodes $D_{0}^{(k)}, \ldots, D_{S}^{(k)}$ are translating the temporal aspect of the model. The traffic covariate is used serve as the dependence link connecting bridges. Traffic dynamics have been monitored and quantified in the Netherlands, for instance (?). The set of bridge-to-bridge edges $\mathcal{E}_{\Theta}^{(\rightarrow, t)}$ is specified through traffic dynamics. A possible layout is shown though in Fig. 4 which captures a distribution of $K$ bridges across a highway section. In this case, for a any time step $t \geq 0$, nodes $\left\{T_{t}^{(k)}\right\}$ and $\left\{T_{t}^{(k+1)}\right\}$ are bonded in a consecutive manner. A pair of bridges are (un)conditionally independent given sets of covariates. This defines the dependence graph structure $\mathcal{G}^{K, S}$; only the conditional distribution set $\mathcal{P}^{K, S}$ has to be specified.

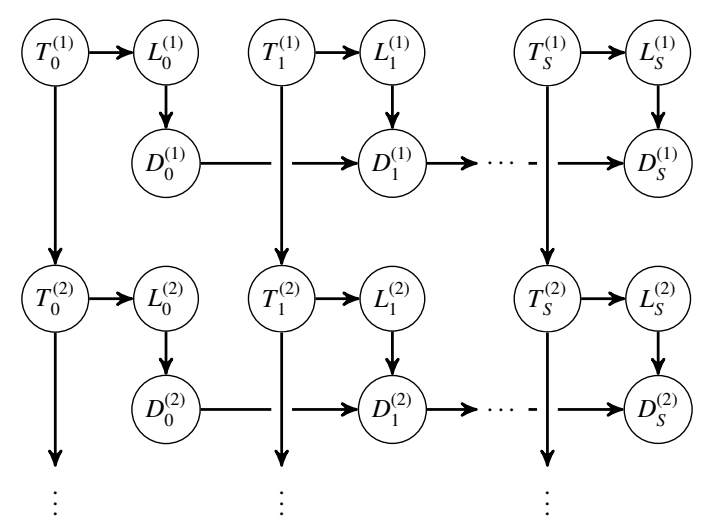

Figure 4: Example layout of covariate-DBN structure $\mathbf{B}^{K, S}$

\subsection{Traffic and load data}

Data on traffic and loading is obtained from a WeighIn-Motion (WIM) system. In ? the same data coming from a WIM installation is input to model multidimensional distribution of axle loads together with other related quantities. A thorough investigation of dependencies between these quantities through a copula repre- sentation is presented. Here WIM data is used to derive a probability distribution on traffic density defined as the number of axles per time over a $100 \mathrm{~m}$ bridge. In addition, the conditional probability distribution of loading given traffic density is derived assuming the covariate-DBN dependence structure presented in the previous subsection. This monitoring installation was set on a two-lane (fast and slow) motorway a few kilometres from a steel bridge in the Netherlands. As only the mechanism of fatigue for orthotropic steel bridges is investigated, loading coming from fluctuating stresses caused by vehicles is in general the most important factor and is seen as a random variable whose distribution is yearly stationary. The nature of traffic intensity influencing the loading behaviour is also stochastic (?). Both distributions of loading and traffic are computed given sample distributions bootstrapped from WIM data. The data is first exploited so that kernel density estimators are computed for fast and slow lanes in a congested traffic configuration. Axles' positions and weights are further obtained by queuing all the vehicles the system recorded over a month. More precisely, a so-called 'train' of vehicles is created. By bootstrapping over a number of fixed vehicles among the total amount of recorded vehicles, a random distribution of vehicles is derived. The generated train provides each fast and slow lane vehicles' separation, axle position and weight, and the number of vehicles per lane. The loading moments are then computed using a finite element method whose discretization step is that of the triangular Bartlett window over the span of the bridge. In this case, the highest loading moment for a vehicle crossing the bridge occurs when it is located halfway through it.

The scatter plot displaying the number of axles against loading and the marginal probability distribution function (PDF) of loading are illustrated in Fig. 5(a) and 5 (b), respectively. Both distributions are plotted conditionally on the number of axles being strictly positive. Equivalently, this means there is always loading on the bridge. Many of the recordings refer to a no-loading scenario, namely $P\left(L_{t}^{(k)}=0\right)=P(\#$ axles $=0)=0.432$, for any $k$. In this configuration, the load variable is discretized by setting the following thresholds which are often used in probabilistic bridge design (ref). A Heavy loaded situation is seen as all the recorded loads lying above the $97^{\text {th }}$ quantile bin of the load PDF conditioned on their being at least one axle. Numerically, this value represents $751.189 \mathrm{kN}$ which can also be written as $P\left(L_{t}^{(k)} \leq 751.189 k N \mid \#\right.$ Axles $\left.>0\right)=0.97$. In Fig. 5(b) the Heavy load is represented by the shaded area below the PDF curve. Similarly, for Normal and Light loading states, values lying in between the $90^{\text {th }}$ 


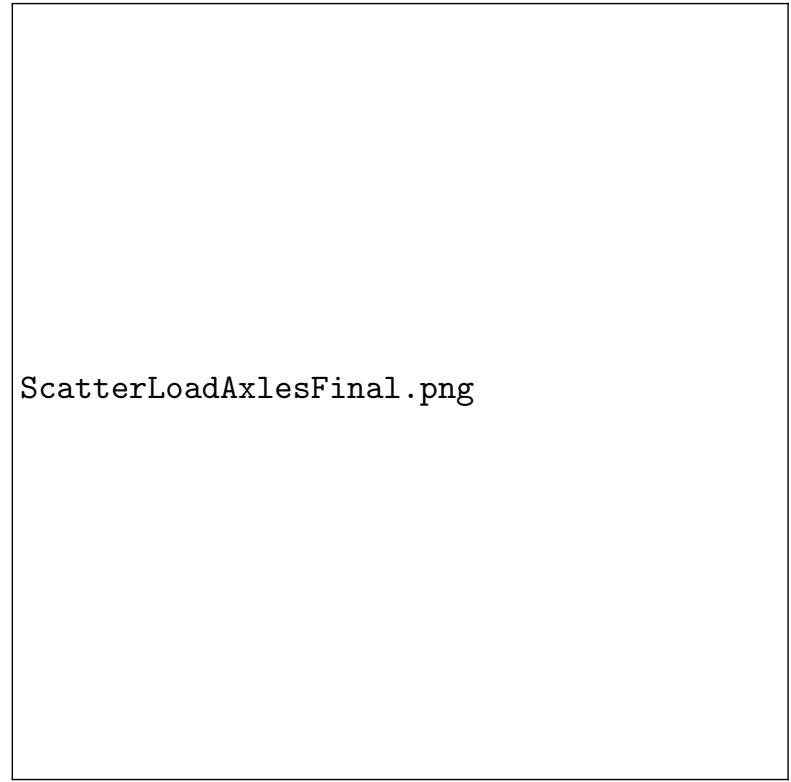

(a) Scatter plot of the number of axles against loading conditionally on \{\#axles $>0\}$

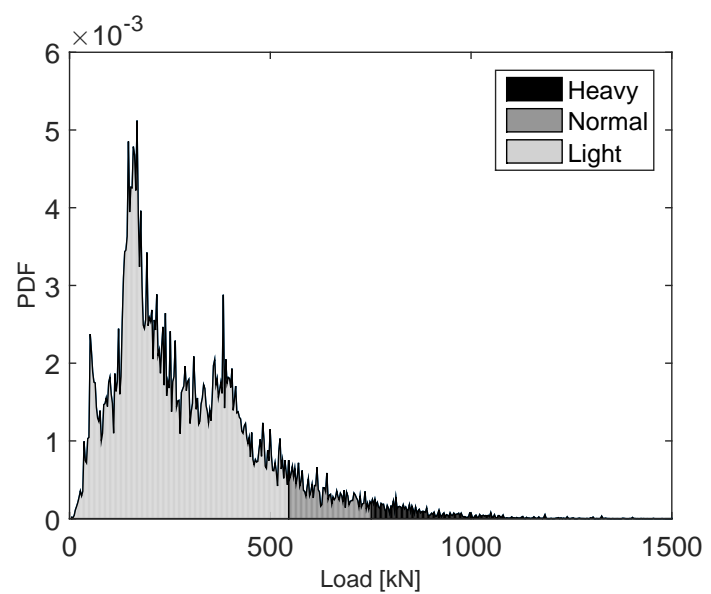

(b) Probability distribution of the total load conditionally on \{\#axles > $0\}$

Figure 5: Load distribution conditionally on $\{$ \#axles $>0\}$

and the $97^{\text {th }}$ quantile bins and below the $90^{\text {th }}$ quantile bin are chosen respectively. These are shown in Fig 5(a) through the dark and light grey scatter points for the Normal and Light loading cases. This way the probability distribution $f_{L_{t}^{(k)} \mid p a\left(L_{t}^{(k)}\right)}$ is fully determined. For every time slice $t$, the quantification of the conditional probability distribution of traffic nodes $\left\{f_{T_{t}^{(k)} \mid p a\left(T_{t}^{(k)}\right)}\right\}_{1 \leq k \leq K}$ was in turn obtained from the National Data Warehouse for Traffic Information (NDW) measurements performed in 2013 from several Dutch highways (?) and broken down into a 3 -state space $\{$ High, Medium, Low $\}$. It is further denoted by $\mathcal{L}=\{$ Heavy, Normal, Light $\}$ and $\mathcal{T}=\{$ High, Medium, Low $\}$ the sets that processes $L_{t}^{(k)}$ and $T_{t}^{(k)}$ take, respectively, value in.

\subsection{Elicitation results}

The complete SEJ experiment is presented in ?. The elicitation was carried out with three experts on steel bridge reliability and management. Particularly, the seed questions refer to historical data on crack length collected between 2006 and 2011 at a highway steel bridge in the Netherlands. A typical seed question asked to the experts is the following:

"An $80 \mathrm{~mm}$ crack was detected located in the deck plate 33 years after construction, what would be its length the following year?"

By varying the time gap between two crack measurements, the age of the bridge at the time of the first measurement, the crack measurement technique as well as the crack location, a total number of 12 seed questions were asked. The remainder of the questionnaire comprises the questions of interest $\mathbf{Q 1}$ and $\mathbf{Q 2}$ which were introduced in section 3.2. They must be asked for each element $k$ (moveable of fixed bridge), loading configuration $\mathcal{L}=\{$ Heavy, Normal, Light $\}$ and type of transition considered $(1 \rightarrow 2,2 \rightarrow 3$ and $3 \rightarrow 4)$, making a total of 24 items of interest. Q1 allows fully calibrating the transition probability matrix as shown in eq. (10) while the second question provides the missing conditional probabilities of node $D_{t}$ given $D_{t-1}$ and $L_{t}$ as the covariate-DBN structure introduced in section 4.2 suggests. From notation introduced in section 2, we have $\left\{f_{D_{t}^{(k)} \mid p a\left(D_{t}^{(k)}\right)}\right\}_{1 \leq k \leq K}$. From the law of total probability, we get

$f_{D_{t}^{(k)}}(x)=\left\{\begin{array}{cc}\sum_{l \in \mathcal{L}} P\left(D_{t}^{(k)}=x \mid L_{t}^{(k)}=l\right) P\left(L_{t}^{(k)}=l\right) & t=0 \\ \sum_{l \in \mathcal{L}} \sum_{y \in\{x, x-1\}} P\left(D_{t}^{(k)}=x \mid D_{t-1}^{(k)}=y, L_{t}^{(k)}=l\right) & \\ \times P\left(L_{t}^{(k)}=l\right) P\left(D_{t-1}^{(k)}=y\right) & \end{array}\right.$ 
In particular, the terms $P\left(D_{t}^{(k)}=x \mid L_{t}^{(k)}=l\right.$ ) (for $t=$ $0)$ and $P\left(D_{t}^{(k)}=x \mid D_{t-1}^{(k)}=x-1, L_{t}^{(k)}=l\right)$ are the ones elicited from Q2. As a consequence, the burden for experts (i.e, the number of queries) increases in the number of states $\Omega$ for the Markov processes $D_{t}^{(k)}$, the number of edges that are incident to the Markov chain $\left(\mathcal{E}_{D^{\llcorner\Theta}}^{(k)}\right)$, and the number of states of the incident covariates.

Using the results in Table 1 by taking the median values $\left(50^{\text {th }}\right.$ percentile) together with eq. (10), the corresponding transition probability matrices for each class of bridge can be derived. Moreover, from eq. (8) and eq. (10), the complete matrix of expected duration of transition can be retrieved as well

$$
\begin{aligned}
\mathbf{P}_{\mathbf{M}} & =\left(\begin{array}{cccc}
0.954 & 0.046 & 0 & 0 \\
0 & 0.905 & 0.095 & 0 \\
0 & 0 & 0.834 & 0.166 \\
0 & 0 & 0 & 1
\end{array}\right), \\
\mathbf{P}_{\mathbf{F}} & =\left(\begin{array}{cccc}
0.976 & 0.024 & 0 & 0 \\
0 & 0.797 & 0.203 & 0 \\
0 & 0 & 0.824 & 0.176 \\
0 & 0 & 0 & 1
\end{array}\right) \\
\mathbf{E}_{\mathbf{M}} & =\left(\begin{array}{cccc}
\infty & 21.62 & 32.14 & 38.16 \\
\infty & \infty & 10.52 & 16.54 \\
\infty & \infty & \infty & 6.02 \\
\infty & \infty & \infty & 1
\end{array}\right), \\
\mathbf{E}_{\mathbf{F}} & =\left(\begin{array}{llll}
\infty & 41.14 & 46.08 & 51.77 \\
\infty & \infty & 4.94 & 10.63 \\
\infty & \infty & \infty & 5.69 \\
\infty & \infty & \infty & 1
\end{array}\right)
\end{aligned}
$$

where subscripts $\mathbf{M}$ and $\mathbf{F}$ denote the moveable and

Table 1: Assessments obtained from the performance based combination scheme (IT) for expected transitions (Yrs) between sequential degradation conditions defined in Table 2 after removing one seed question

\begin{tabular}{ccccc} 
Bridge type & Transition & $5^{\text {th }}$ & $50^{\text {th }}$ & $95^{\text {th }}$ \\
\hline \multirow{4}{*}{ Moveable } & $1 \rightarrow 2$ & 3.09 & 21.62 & 49.45 \\
& $2 \rightarrow 3$ & 5.04 & 10.52 & 24.59 \\
& $3 \rightarrow 4$ & 3.30 & 6.02 & 28.18 \\
\hline \multirow{3}{*}{ Fixed } & $1 \rightarrow 2$ & 4.73 & 41.14 & 54.60 \\
& $2 \rightarrow 3$ & 3.81 & 4.94 & 20.25 \\
& $3 \rightarrow 4$ & 1.15 & 5.69 & 34.56 \\
\hline
\end{tabular}

fixed classes, respectively. Backward reasoning also applies, that is, conditioning on one or more states of the covariates, the conditional transition probability matrix can be computed as well as the conditional expectation matrix using eq. (8). Upon this basis, the annual probability distribution of process $D_{t}$ to reach the worst state, $P\left(D_{t}=4 \mid D_{0}=1\right)$ (eq. (11)), using the IT DM combined distribution are displayed in Fig. 6 for both moveable and fixed bridge categories. For each distribution the median ( $50^{\text {th }}$ quantile) is presented. The differences in sensitiveness through inserted information highlighted by the posterior distributions are quite sharp. Unlike the case featuring a Normal load, observe that distributions conditioned on a heavy load do not differ much between the two classes of bridge considered.

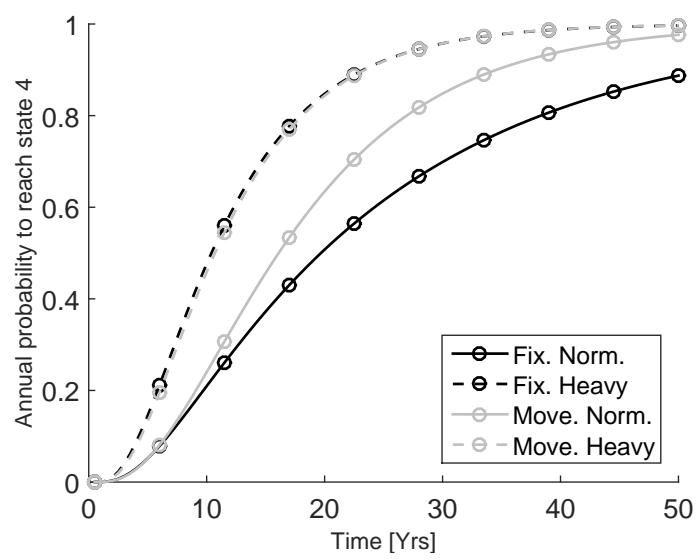

Figure 6: Performance based combination of the median estimate for annual probability distribution to reach worst state (see Table 2) for both Moveable and Fixed bridges classes.

\section{Numerical experiment}

Various experiments are presented showing the sensitivity of the proposed model for the network-scale extension using the methodology presented in Section 2.4. Note that the algorithm used for inference here is based on the paper of ? that is implemented in the Bayesian network framework Smile application programming interface (API). It is shown how much the network beliefs are modified when information is obtained from various covariates and elements at different points in time. As previously discussed, this can lead to prohibitive number of combinations. Scenarios leading to changing traffic conditions are numerous as well as their loading characteristics. Examples affecting traffic conditions include maintenance for one or more bridges in the surrounding network area, traffic accidents or environmental disasters. 
Consider a single 4-state condition space for both bridge categories (fixed and moveable) whose conditions are defined in Table 2.

A hypothetical network of bridges is constructed using the new covariate-DBN model introduced in section 2.3. The quantification methods used for the conditional probability sets are those introduced in sections 4.2 and 4.3. The network is comprised of four bridges, three moveable and one fixed, whose layout is similar to that of Fig. 4 having the same set of covariates $\Theta_{t}^{(k)}=\left\{T_{t}^{(k)}, L_{t}^{(k)}\right\}$ standing for traffic density and loading. The example related to bridges located at the intersection highways A2 and A15 in the Netherlands. A15 has one of the most dense yearly traffic while A2 is more average (?). We feel that such a configuration resembles many real-world cases. For the example, bridges 1 (fixed) and 2 belong to A15 and bridges 3 and 4 to A2.

A first example of inference is illustrated in Fig. 7 where the CDF of the condition states for Bridges 1 and 3 are plotted at each time step. The left column is the unconditional (NOI) case and right column shows information on High traffic inserted for Bridge 1 consecutively in each year from 5 to 10 . Observe that probability area for state 4 has increased (right column) for both bridges with respect to the unconditional case. Recall that Bridge 1 belongs to a highway whose traffic density (give distribution) is expected to be higher than that of Bridge 3 (distribution). This shows that the distribution of Bridge 3 is relatively sensitive to information obtained from Bridge 1. Moreover, inserting information that deviates significantly from the expected should have a greater impact on the sensitivity. The outcome of inference is mainly governed by the conditional probability distributions across traffic nodes obtained from ?. For instance, the conditional distribution $T_{t}^{(3)} \mid T_{t}^{(2)}$ is given in Table 3 . The same tests were carried out using Low and Medium states individually in the same context and updated distributions showed minor modification. Similar observations were also drawn with respect to Bridges 2 and 4. Fig. 7 showed the cumulative effects of inserting hight traffic information into the network. The effect of inserting high traffic information individually as shown in Fig 8 are examined. The horizontal axis denotes $\left(\mu_{\omega}^{(k)}\right)$ the vertical axis the sensitivity measure $\sigma_{i, \Theta}$ computed as defined in eq. (5) for state $i=4$. Each plot represents a fixed time slice $t \in\{5,10, \ldots, S=50\}$, the boldest curve represents $t=5$ and lightest curve refers to $t=50$. Thus, the " $t=5$ " curve represents the sensitivity at $t=5$ of inserting hight traffic information individually over the time horizon. Notice that once information has been inserted posteriorly to the fixed time epoch $\left(\mu_{\omega}^{(k)}>t\right)$, the sensitivity drops to zero as previously detailed in eq (6). The sensitiveness dramatically decreases both as information is inserted later in time and evaluated later in time. Thus, the figure shows that it is most relevant to insert information as early as possible and the return on information dramatically decreases over time. The same comments can be made for Bridge 3 and more generally shows a lesser amplitude for the $\sigma_{4, \Theta}$ curves. For example, $\mu_{\omega}^{(k)}$ peaks at $\sim 18 \%$ for Bridge 1 , while the maximum does not reach $14 \%$ for Bridge 3 . This reduced sensitivity is to be expected as it has a downstream impact from where the information was directly obtained Bridge 1. Similar tests were performed for the remainder of the network, namely Bridges 2 and 4, which showed similar behavior.

Likewise, analyses on $\eta_{\omega}^{(k)}$ were performed as defined in section 2.4 for cumulative information. Sensitivity curves for $\eta_{\omega}^{(k)}$ are displayed Fig. 9. The various grey gradient curves read in similar fashion to those of the plots displayed in Fig 8. Compared to the single insertion case (Fig. 7), the sensitivity increases dramatically for every fixed time epoch. This is evidenced by comparing the ' $t=5$ ' curves; the sensitivity for Fig. 9 peaks above $120 \%$ whereas Fig. 7 (Bridge 1) does not pass $20 \%$. Most importantly, the figure demonstrates that more information is always better and information loses its value over time. The latter can be explained by the distribution of each state being bounded asymptotically by some upper and lower conditional distributions as shown in Fig. 6. In the case of most or least expected information being inserted consecutively from $t=0$ throughout the network, the degradation distribution will correspond to respective bounding distribution. In this numerical experiment, the upper bound corresponds to the least expected information (i.e., high traffic/heavy loading) being inserted. Although, not demonstrated from the experiment, we believe that regardless of the manner information is inserted (i.e., consecutive or not), more information will always have a greater impact on sensitivity.

\section{Conclusions}

An extension to the classic dynamic Bayesian network framework which is termed the covariate-DBN is proposed. a second dimension for $K$ elements is added as well as method for indirectly linking them through a set of covariates. It is further proposed a Markov chain as the underlying stochastic process for the covariateDBN. In the case where limited data is available, a formal mathematical framework is developed making use of Cooke's method for structured expert judgement to 


\begin{tabular}{ll} 
State & Definition \\
\hline 1 - Excellent & Almost no damage/cracks are present. A new bridge is assumed to start from this state. \\
2 - Fair & At least one crack in the deck plate that can be detected ultrasonically [30mm, 100mm] \\
3 - Mediocre & Multiple cracks are present [30mm, 500mm]; at least one crack requires repair \\
4 - Poor & $\begin{array}{l}\text { Multiple significant fatigue cracks with at least one }>500 \mathrm{~mm} \text { in the deck plate that needs urgent } \\
\text { repair; this condition does not mean a collapse but a threat to safety and/or functionality. }\end{array}$
\end{tabular}
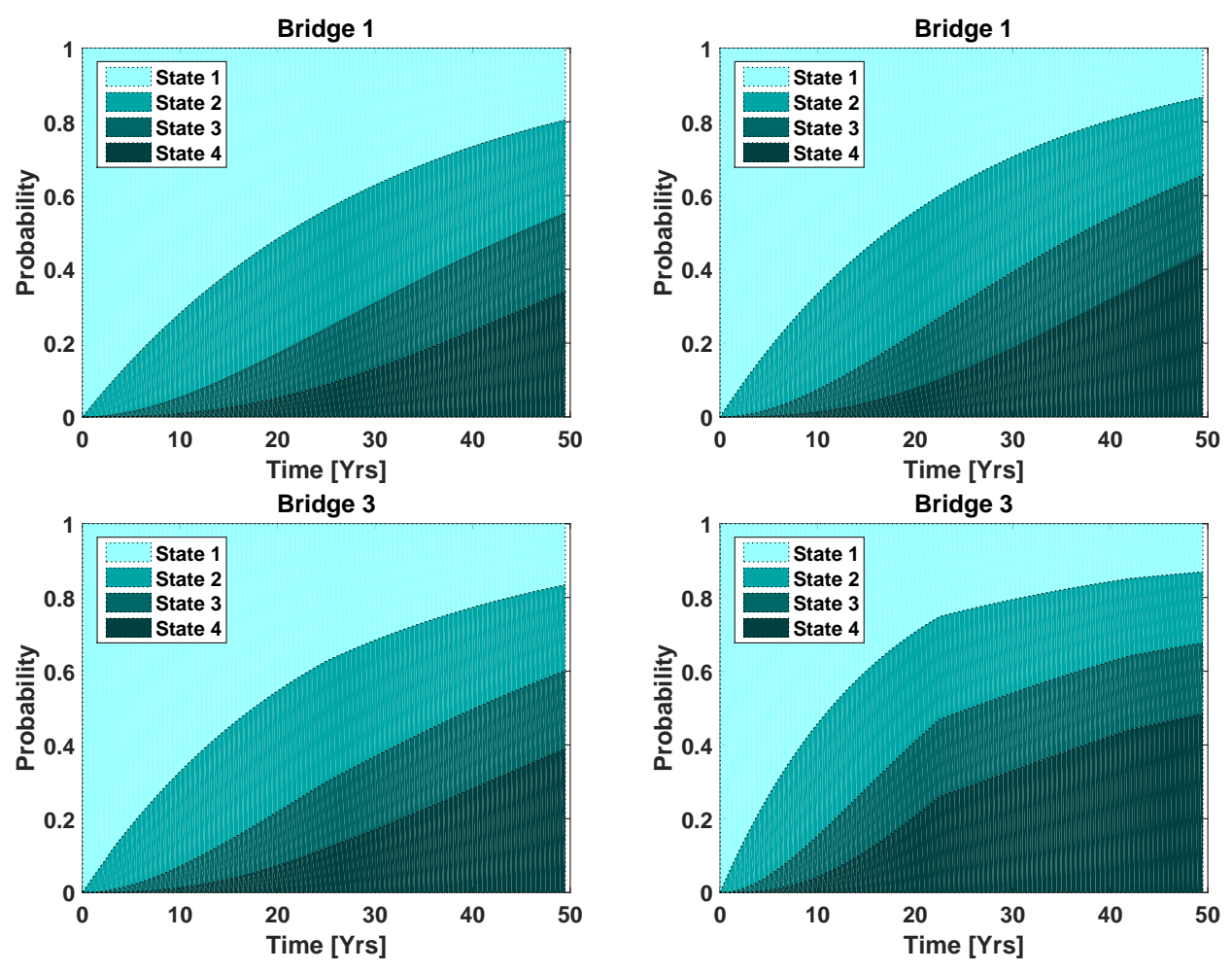

Figure 7: Impact of High Traffic Information on the Network

Table 3: Conditional probability distribution of traffic process $T_{t}^{(3)}$ given $T_{t}^{(2)}$

\begin{tabular}{lccc}
$T_{t}^{(3)} \mid T_{t}^{(2)}$ & Low & Medium & High \\
\hline Low & 0.934 & 0.0448 & 0.0385 \\
Medium & 0.0492 & 0.879 & 0.0651 \\
High & 0.0168 & 0.0762 & 0.8964 \\
\hline
\end{tabular}

parametrize a Markov chain and the covariate relationships between elements in the covariate-DBN. Some metrics are also presented for evaluating the sensitivity of information inserted into the covariate-DBN.

The proposal is then applied to a real-world bridge network application based on steel bridges in the Netherlands. It is shown how traffic and load informa- tion may serve as covariates to link bridge elements in the covariate-DBN. An actual expert judgment elicitation was carried out to parametrize the model using the prescribed methods. Numerical experiments show that information is most valuable as early as possible, and the value of information decreases over time.

While the model is applied to a specific bridge network scenario, different sets of covariates could be envisioned in the same framework. Furthermore, we believe the model could be expanded to other bridge types and civil infrastructure. Applications are not only limited to degradation modelling but could include other fields and contexts such as financial asset modelling and disease propagation.

In sections 2.4 and 5, it is shown how one could reduce the computational intractability referring to run- 
Bridge 1

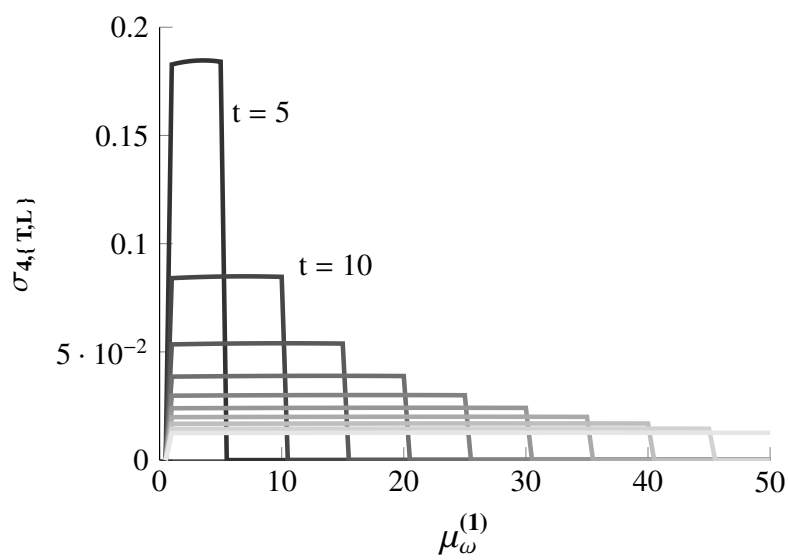

Bridge 3

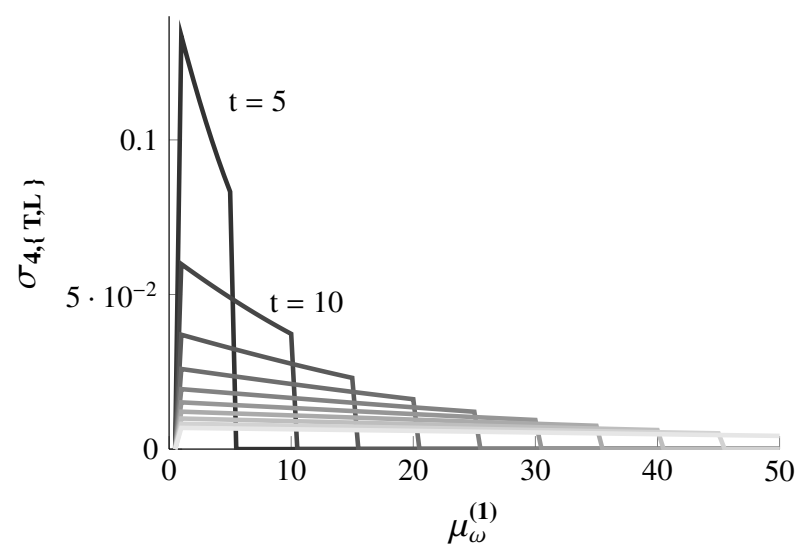

Figure 8: Sensitivity curves for $\sigma_{4, \Theta}$ plotted against $m_{\omega}^{(1)}$ where the colour gradient from dark to light grey for each curve indicates fixed time epochs for each plot spaced by 5 years for bridge 1 (left) and bridge 3 (right).

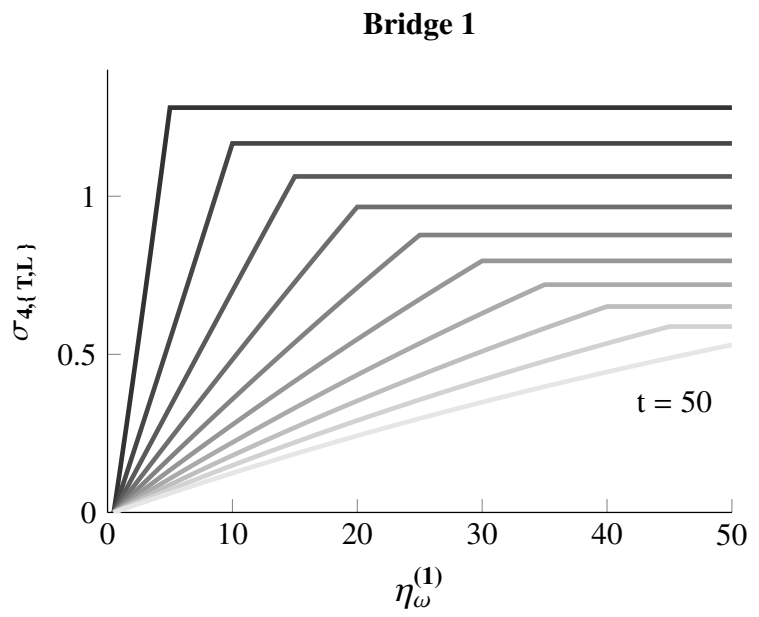

Figure 9: Sensitivity curves for $\sigma_{4, \Theta}$ (left) where the colour gradient from dark to light grey for each curve indicates fixed time epochs for each plot spaced by 5 years. The type of information inserted is state High for node $T_{t}^{(1)}$

ning through all the possible combinations of inference. In particular, from figures 8 and 9 it is observed that :

- cumulative inserted pieces of information dominate over individual piece of information; in other words, any inference combination having a lower number of inserted pieces of information than its cumulative counterpart will show a less sensitive change in the posterior distribution. Practically speaking, continuous monitoring should prevail as opposed to condition-based (by also taking into account cost constraints)
- the sensitiveness of the inserted information decreases in time so that pieces of evidence inserted at early epochs should be preferred over later ones. This means that if significant and unexpected event are observed (represented by the type of inserted information), the sensitivity metric is also able to capture those.

Thus, by advantageously combining the two above observations, one could selectively opts for the most sensitive combinations of inference. This further results in substantially decreasing the inference choices.

For discrete BNs the main limitation of the proposed methodology refers to dimensionality. Our model further increases this complexity through the added $k$ dimension. Other classes of BNs dealing with continuous distributions could facilitate the parametrization procedure. For example, a dynamic non-parametric class recently developed (?) could be a useful tool to overcome this. An extension to influence diagrams would provide a decision making framework for the underlying covariate-DBN to facilitate managers applying model forecasts. 